\title{
Research Article \\ Generalizations of the Nash Equilibrium Theorem in the KKM Theory
}

\author{
Sehie Park ${ }^{1,2}$ \\ ${ }^{1}$ The National Academy of Sciences, Seoul 137-044, Republic of Korea \\ ${ }^{2}$ Department of Mathematical Sciences, Seoul National University, Seoul 151-747, Republic of Korea
}

Correspondence should be addressed to Sehie Park, shpark@math.snu.ac.kr

Received 5 December 2009; Accepted 2 February 2010

Academic Editor: Anthony To Ming Lau

Copyright $@ 2010$ Sehie Park. This is an open access article distributed under the Creative Commons Attribution License, which permits unrestricted use, distribution, and reproduction in any medium, provided the original work is properly cited.

The partial KKM principle for an abstract convex space is an abstract form of the classical KKM theorem. In this paper, we derive generalized forms of the Ky Fan minimax inequality, the von Neumann-Sion minimax theorem, the von Neumann-Fan intersection theorem, the Fan-type analytic alternative, and the Nash equilibrium theorem for abstract convex spaces satisfying the partial KKM principle. These results are compared with previously known cases for G-convex spaces. Consequently, our results unify and generalize most of previously known particular cases of the same nature. Finally, we add some detailed historical remarks on related topics.

\section{Introduction}

In 1928, John von Neumann found his celebrated minimax theorem [1] and, in 1937, his intersection lemma [2], which was intended to establish easily his minimax theorem and his theorem on optimal balanced growth paths. In 1941, Kakutani [3] obtained a fixed point theorem for multimaps, from which von Neumann's minimax theorem and intersection lemma were easily deduced.

In 1950, John Nash [4, 5] established his celebrated equilibrium theorem by applying the Brouwer or the Kakutani fixed point theorem. In 1952, Fan [6] and Glicksberg [7] extended Kakutani's theorem to locally convex Hausdorff topological vector spaces, and Fan generalized the von Neumann intersection lemma by applying his own fixed point theorem. In 1972, Himmelberg [8] obtained two generalizations of Fan's fixed point theorem [6] and applied them to generalize the von Neumann minimax theorem by following Kakutani's method in [3].

In 1961, Ky Fan [9] obtained his KKM lemma and, in 1964 [10], applied it to another intersection theorem for a finite family of sets having convex sections. This was applied in 1966 [11] to a proof of the Nash equilibrium theorem. This is the origin of the application of the KKM theory to the Nash theorem. In 1969, Ma [12] extended Fan's intersection theorem 
[10] to infinite families and applied it to an analytic formulation of Fan type and to the Nash theorem for arbitrary families.

Note that all of the above results are mainly concerned with convex subsets of topological vector spaces; see Granas [13]. Later, many authors tried to generalize them to various types of abstract convex spaces. The present author also extended them in our previous works [14-28] in various directions. In fact, the author had developed theory of generalized convex spaces (simply, G-convex spaces) related to the KKM theory and analytical fixed point theory. In the framework of G-convex spaces, we obtained some minimax theorems and the Nash equilibrium theorems in our previous works [17, 18, 21, 22], based on coincidence theorems or intersection theorems for finite families of sets, and in [22], based on continuous selection theorems for the Fan-Browder maps.

In our recent works [24-26], we studied the foundations of the KKM theory on abstract convex spaces. The partial KKM principle for an abstract convex space is an abstract form of the classical KKM theorem. A KKM space is an abstract convex space satisfying the partial KKM principle and its "open" version. We noticed that many important results in the KKM theory are closely related to KKM spaces or spaces satisfying the partial KKM principle. Moreover, a number of such results are equivalent to each other.

On the other hand, some other authors studied particular types of KKM spaces and deduced some Nash-type equilibrium theorem from the corresponding partial KKM principle, for example, [17, 21, 29-33], explicitly, and many more in the literature, implicitly. Therefore, in order to avoid unnecessary repetitions for each particular type of KKM spaces, it would be necessary to state clearly them for spaces satisfying the partial KKM principle. This was simply done in [27].

In this paper, we study several stages of such developments from the KKM principle to the Nash theorem and related results within the frame of the KKM theory of abstract convex spaces. In fact, we clearly show that a sequence of statements from the partial KKM principle to the Nash equilibria can be obtained for any space satisfying the partial KKM principle. This unifies previously known several proper examples of such sequences for particular types of KKM spaces. More precisely, our aim in this paper is to obtain generalized forms of the KKM space versions of known results due to von Neumann, Sion, Nash, Fan, Ma, and many followers. These results are mainly obtained by (1) fixed point method, (2) continuous selection method, or (3) the KKM method. In this paper, we follow method (3) and will compare our results to corresponding ones already obtained by method (2).

In Section 2, we state basic facts and examples of abstract convex spaces in our previous works [24-26]. Section 3 deals with a characterization of the partial KKM principle and shows that such principle is equivalent to the generalized Fan-Browder fixed point theorem. In Section 4, we deduce a general Fan-type minimax inequality from the partial KKM principle. Section 5 deals with various von Neumann-Sion-type minimax theorems for abstract convex spaces.

In Section 6, a collective fixed point theorem is deduced as a generalization of the FanBrowder fixed point theorem. Section 7 deals with the Fan-type intersection theorems for sets with convex sections in product abstract convex spaces satisfying the partial KKM principle. In Section 8, we deduce a Fan-type analytic alternative and its consequences. Section 9 is devoted to various generalizations of the Nash equilibrium theorem and their consequences. Finally, in Section 10, some known results related to the Nash theorem and historical remarks are added.

This paper is a revised and extended version of [22, 27] and a supplement to [24-26], where some other topics on abstract convex spaces can be found. 


\section{Abstract Convex Spaces and the KKM Spaces}

Multimaps are also called simply maps. Let $\langle D\rangle$ denote the set of all nonempty finite subsets of a set $D$. Recall the following in [24-26].

Definition 2.1. An abstract convex space $(E, D ; \Gamma)$ consists of a topological space $E$, a nonempty set $D$, and a multimap $\Gamma:\langle D\rangle \multimap E$ with nonempty values $\Gamma_{A}:=\Gamma(A)$ for $A \in\langle D\rangle$.

For any $D^{\prime} \subset D$, the $\Gamma$-convex hull of $D^{\prime}$ is denoted and defined by

$$
\operatorname{co}_{\Gamma} D^{\prime}:=\bigcup\left\{\Gamma_{A} \mid A \in\left\langle D^{\prime}\right\rangle\right\} \subset E .
$$

A subset $X$ of $E$ is called a $\Gamma$-convex subset of $(E, D ; \Gamma)$ relative to $D^{\prime}$ if for any $N \in\left\langle D^{\prime}\right\rangle$ we have that $\Gamma_{N} \subset X$, that is, $\operatorname{co}_{\Gamma} D^{\prime} \subset X$.

When $D \subset E$, the space is denoted by $(E \supset D ; \Gamma)$. In such case, a subset $X$ of $E$ is said to be $\Gamma$-convex if $\operatorname{co}_{\Gamma}(X \cap D) \subset X$; in other words, $X$ is $\Gamma$-convex relative to $D^{\prime}:=X \cap D$. In case $E=D$, let $(E ; \Gamma):=(E, E ; \Gamma)$.

Example 2.2. The following are known examples of abstract convex spaces.

(1) A triple $\left(\Delta_{n} \supset V\right.$; co) is given for the original KKM theorem [34], where $\Delta_{n}$ is the standard $n$-simplex, $V$ is the set of its vertices $\left\{e_{i}\right\}_{i=0}^{n}$, and co: $\langle V\rangle \multimap \Delta_{n}$ is the convex hull operation.

(2) A triple $(X \supset D ; \Gamma)$ is given, where $X$ and $D$ are subsets of a t.v.s. $E$ such that co $D \subset X$ and $\Gamma:=$ co. Fan's celebrated KKM lemma [9] is for ( $E \supset D ; c 0)$.

(3) A convex space $(X \supset D ; \Gamma)$ is a triple where $X$ is a subset of a vector space such that co $D \subset X$, and each $\Gamma_{A}$ is the convex hull of $A \in\langle D\rangle$ equipped with the Euclidean topology. This concept generalizes the one due to Lassonde for $X=D$; see [35]. However he obtained several KKM-type theorems w.r.t. $(X \supset D ; \Gamma)$.

(4) A triple $(X \supset D ; \Gamma)$, is called an $H$-space if $X$ is a topological space and $\Gamma=\left\{\Gamma_{A}\right\}$ is a family of contractible (or, more generally, $\omega$-connected) subsets of $X$ indexed by $A \in\langle D\rangle$ such that $\Gamma_{A} \subset \Gamma_{B}$ whenever $A \subset B \in\langle D\rangle$. If $D=X$, then $(X ; \Gamma):=(X, X ; \Gamma)$ is called a $c$-space by Horvath $[36,37]$.

(5) Hyperconvex metric spaces due to Aronszajn and Panitchpakdi are particular cases of $c$-spaces; see [37].

(6) Hyperbolic spaces due to Reich and Shafrir [38] are also particular cases of $c$-spaces. This class of metric spaces contains all normed vector spaces, all Hadamard manifolds, the Hilbert ball with the hyperbolic metric, and others. Note that an arbitrary product of hyperbolic spaces is also hyperbolic.

(7) Any topological semilattice $(X, \leq)$ with path-connected interval is introduced by Horvath and Llinares [39].

(8) A generalized convex space or a G-convex space $(X, D ; \Gamma)$ due to Park is an abstract convex space such that for each $A \in\langle D\rangle$ with the cardinality $|A|=n+1$ there exists a continuous function $\phi_{A}: \Delta_{n} \rightarrow \Gamma(A)$ such that $J \in\langle A\rangle$ implies that $\phi_{A}\left(\Delta_{J}\right) \subset \Gamma(J)$.

Here, $\Delta_{J}$ is the face of $\Delta_{n}$ corresponding to $J \in\langle A\rangle$, that is, if $A=\left\{a_{0}, a_{1}, \ldots, a_{n}\right\}$ and $J=\left\{a_{i_{0}}, a_{i_{1}}, \ldots, a_{i_{k}}\right\} \subset A$, then $\Delta_{J}=\operatorname{co}\left\{e_{i_{0}}, e_{i_{1}}, \ldots, e_{i_{k}}\right\}$.

For details, see references of $[17,21,22,40-42]$.

(9) A $\phi_{A}$-space $\left(X, D ;\left\{\phi_{A}\right\}_{A \in\langle D\rangle}\right)$ consists of a topological space $X$, a nonempty set $D$, and a family of continuous functions $\phi_{A}: \Delta_{n} \rightarrow X$ (that is, singular $n$-simplexes) for $A \in\langle D\rangle$ with $|A|=n+1$. Every $\phi_{A}$-space can be made into a G-convex space; see [43]. 
Recently $\phi_{A}$-spaces are called GFC-spaces in [44] and FC-spaces [43] or simplicial spaces [45] when $X=D$.

(10) Suppose that $X$ is a closed convex subset of a complete $\mathbb{R}$-tree $H$, and for each $A \in\langle X\rangle, \Gamma_{A}:=\operatorname{conv}_{H}(A)$, where $\operatorname{conv}_{H}(A)$ is the intersection of all closed convex subsets of $H$ that contain $A$; see Kirk and Panyanak [46]. Then $(H \supset X ; \Gamma)$ is an abstract convex space. example.

(11) A topological space $X$ with a convexity in the sense of Horvath [47] is another

(12) A $\mathbb{B}$-space due to Briec and Horvath [30] is an abstract convex space.

Note that each of (2)-(12) has a large number of concrete examples and that all examples (1)-(9) are G-convex spaces.

Definition 2.3. Let $(E, D ; \Gamma)$ be an abstract convex space. If a multimap $G: D \multimap E$ satisfies

$$
\Gamma_{A} \subset G(A):=\bigcup_{y \in A} G(y) \quad \forall A \in\langle D\rangle,
$$

then $G$ is called a KKM map.

Definition 2.4. The partial KKM principle for an abstract convex space $(E, D ; \Gamma)$ is the statement that, for any closed-valued KKM map $G: D \multimap E$, the family $\{G(y)\}_{y \in D}$ has the finite intersection property. The KKM principle is the statement that the same property also holds for any open-valued KKM map.

An abstract convex space is called a KKM space if it satisfies the KKM principle.

In our recent works [24-26], we studied the foundations of the KKM theory on abstract convex spaces and noticed that many important results therein are related to the partial KKM principle.

Example 2.5. We give examples of KKM spaces as follows.

(1) Every G-convex space is a KKM space [18].

(2) A connected linearly ordered space $(X, \leq)$ can be made into a KKM space [26].

(3) The extended long line $L^{*}$ is a KKM space $\left(L^{*}, D ; \Gamma\right)$ with the ordinal space $D:=$ $[0, \Omega]$; see [26]. But $L^{*}$ is not a $G$-convex space.

(4) For a closed convex subset $X$ of a complete $\mathbb{R}$-tree $H$, and $\Gamma_{A}:=\operatorname{conv}_{H}(A)$ for each $A \in\langle X\rangle$, the triple $(H \supset X ; \Gamma)$ satisfies the partial KKM principle; see [46]. Later we found that $(H \supset X ; \Gamma)$ is a KKM space [48].

(5) Horvath's convex space $(X ; \Gamma)$ with the weak Van de Vel property is a KKM space, where $\Gamma_{A}:=[[A]]$ for each $A \in\langle X\rangle$; see $[47,48]$.

(6) A $\mathbb{B}$-space due to Briec and Horvath [30] is a KKM space.

Now we have the following diagram for triples $(E, D ; \Gamma)$ :

$$
\begin{gathered}
\text { simplex } \Longrightarrow \text { convex subset of a t.v.s. } \Longrightarrow \text { Lassonde-type convex space } \\
\Longrightarrow H \text {-space } \Longrightarrow \text { G-convex space } \Longleftrightarrow \phi_{A} \text {-space } \Longrightarrow \text { KKM space } \\
\Longrightarrow \text { space satisfying the partial KKM principle } \\
\Longrightarrow \text { abstract convex space. }
\end{gathered}
$$

It is not known yet whether there is a space satisfying the partial KKM principle that is not a KKM space. 


\section{The KKM Principle and the Fan-Browder Map}

Let $(E, D ; \Gamma)$ be an abstract convex space.

Recall the following equivalent form of [26, Theorem 8.2].

Theorem 3.1. Suppose that $(E, D ; \Gamma)$ satisfies the partial KKM principle and a map $G: D \multimap E$ satisfies the following.

(1.1) $G$ is closed valued.

(1.2) $G$ is a KKM map (i.e., $\Gamma_{A} \subset G(A)$ for all $A \in\langle D\rangle$ ).

(1.3) There exists a nonempty compact subset $K$ of $E$ such that one of the following holds:

(i) $K=E$,

(ii) $K=\bigcap\{G(z) \mid z \in M\}$ for some $M \in\langle D\rangle$,

(iii) for each $N \in\langle D\rangle$, there exists a compact $\Gamma$-convex subset $L_{N}$ of $E$ relative to some $D^{\prime} \subset D$ such that $N \subset D^{\prime}$ and

$$
L_{N} \cap \bigcap_{z \in D^{\prime}} G(z) \subset K
$$

Then $K \cap \bigcap\{G(z) \mid z \in D\} \neq \emptyset$.

Remark 3.2. Conditions (i)-(iii) in (1.3) are called compactness conditions or coercivity conditions. In this paper, we mainly adopt simply $(\mathrm{i})$, that is, $(E, D ; \Gamma)$ is compact. However, most of results can be reformulated to the ones adopting (ii) or (iii).

Definition 3.3. For a topological space $X$ and an abstract convex space $(E, D ; \Gamma)$, a multimap $T: X \multimap E$ is called a $\Phi$-map or a Fan-Browder map provided that there exists a multimap $S: X \multimap D$ satisfying the follwing:

(a) for each $x \in X, \operatorname{co}_{\Gamma} S(x) \subset T(x)$ (i.e., $N \in\langle S(x)\rangle$ implies that $\Gamma_{N} \subset T(x)$ ),

(b) $X=\bigcup_{z \in M} \operatorname{Int} S^{-}(z)$ for some $M \in\langle D\rangle$. $z \in S(x)\}$.

Here, Int denotes the interior with respect to $E$ and, for each $z \in D, S^{-}(z):=\{x \in X \mid$

There are several equivalent formulations of the partial KKM principle; see [26]. For example, it is equivalent to the Fan-Browder-type fixed point theorem as follows.

Theorem 3.4 (see [26]). An abstract convex space $(E, D ; \Gamma)$ satisfies the partial KKM principle if and only if any $\Phi$-map $T: E \multimap E$ has a fixed point $x_{0} \in E$, that is, $x_{0} \in T\left(x_{0}\right)$.

The following is known.

Lemma 3.5. Let $\left\{\left(X_{i}, D_{i} ; \Gamma_{i}\right)\right\}_{i \in I}$ be any family of abstract convex spaces. Let $X:=\prod_{i \in I} X_{i}$ be equipped with the product topology and $D=\prod_{i \in I} D_{i}$. For each $i \in I$, let $\pi_{i}: D \rightarrow D_{i}$ be the projection. For each $A \in\langle D\rangle$, define $\Gamma(A):=\prod_{i \in I} \Gamma_{i}\left(\pi_{i}(A)\right)$. Then $(X, D ; \Gamma)$ is an abstract convex space.

Let $\left\{\left(X_{i}, D_{i} ; \Gamma_{i}\right)\right\}_{i \in I}$ be a family of $G$-convex spaces. Then $(X, D ; \Gamma)$ is a $G$-convex space. 
It is not known yet whether this holds for KKM spaces.

From now on, for simplicity, we are mainly concerned with compact abstract convex spaces $(E ; \Gamma)$ satisfying the partial KKM principle. For example, any compact $G$-convex space, any compact $H$-space, or any compact convex space is such a space.

\section{The Fan-Type Minimax Inequalities}

Recall that an extended real-valued function $f: X \rightarrow \overline{\mathbb{R}}$, where $X$ is a topological space, is lower [resp., upper] semicontinuous (1.s.c.) (resp., u.s.c.) if $\{x \in X \mid f(x)>r\}$ (resp., $\{x \in X \mid$ $f(x)<r\})$ is open for each $r \in \mathbb{R}$.

For an abstract convex space $(E \supset D ; \Gamma)$, an extended real-valued function $f: E \rightarrow \overline{\mathbb{R}}$ is said to be quasiconcave (resp., quasiconvex) if $\{x \in E \mid f(x)>r\}$ (resp., $\{x \in E \mid f(x)<r\}$ ) is $\Gamma$-convex for each $r \in \overline{\mathbb{R}}$.

From the partial KKM principle we can deduce a very general version of the Ky Fan minimax inequality as follows.

Theorem 4.1. Let $(X, D ; \Gamma)$ be an abstract convex space satisfying the partial KKM principle, $f$ : $D \times X \rightarrow \overline{\mathbb{R}}, g: X \times X \rightarrow \overline{\mathbb{R}}$ extended real functions, and $\gamma \in \overline{\mathbb{R}}$ such that

(3.1) for each $z \in D,\{y \in X \mid f(z, y) \leq \gamma\}$ is closed,

(3.2) for each $y \in X, \operatorname{co}_{\Gamma}\{z \in D \mid f(z, y)>\gamma\} \subset\{x \in X \mid g(x, y)>\gamma\}$,

(3.3) the compactness condition (1.3) holds for $G(z):=\{y \in X \mid f(z, y) \leq \gamma\}$.

Then either (i) there exists $a \hat{x} \in X$ such that $f(z, \hat{x}) \leq \gamma$ for all $z \in D$ or (ii) there exists an $x_{0} \in X$ such that $g\left(x_{0}, x_{0}\right)>r$.

Proof. Let $G: D \multimap X$ be a map defined by $G(z):=\{y \in X \mid f(z, y) \leq \gamma\}$ for $z \in D$. Then each $G(z)$ is closed by (3.1).

Case (i): $G$ is a KKM map.

By Theorem 3.1, we have $\bigcap_{z \in D} G(z) \neq \emptyset$. Hence, there exists a $\widehat{x} \in X$ such that $\widehat{x} \in G(z)$ for all $z \in D$, that is, $f(z, \widehat{x}) \leq \gamma$ for all $z \in D$.

Case (ii): $G$ is not a KKM map.

Then there exists $N \in\langle D\rangle$ such that $\Gamma_{N} \not \subset \bigcup_{z \in N} G(z)$. Hence there exists an $x_{0} \in \Gamma_{N}$ such that $x_{0} \notin G(z)$ for each $z \in N$, or equivalently $f\left(z, x_{0}\right)>\gamma$ for each $z \in N$. Since $\{z \in$ $\left.D \mid f\left(z, x_{0}\right)>\gamma\right\}$ contains $N$, by (3.2), we have $x_{0} \in \Gamma_{N} \subset\left\{x \in X \mid g\left(x, x_{0}\right)>\gamma\right\}$, and hence, $g\left(x_{0}, x_{0}\right)>r$.

Corollary 4.2. Under the hypothesis of Theorem 4.1, let $\gamma:=\sup _{x \in X} g(x, x)$. Then

$$
\inf _{y \in X} \sup _{z \in D} f(z, y) \leq \sup _{x \in X} g(x, x)
$$

Example 4.3. (1) For a compact convex subset $X=D$ of a t.v.s. and $f=g$, if $f(\cdot, y)$ is quasiconcave, then (3.2) holds; and if $f(x, \cdot)$ is 1.s.c., then (3.1) holds. Therefore, Corollary 4.2 generalizes the Ky Fan minimax inequality [49].

(2) For a convex space $X=D$ and $f=g$, Corollary 4.2 reduces to Cho et al. [50, Theorem 9]. 
(3) There is a very large number of generalizations of the Fan minimax inequality for convex spaces, $H$-spaces, $G$-convex spaces, and others. These would be particular forms of Corollary 4.2. For example, see Park [18, Theorem 11], where $(X, D ; \Gamma)$ is a $G$-convex space.

(4) Some particular versions of Corollary 4.2 were given in [27].

\section{The von Neumann-Sion-Type Minimax Theorems}

Let $\left(X ; \Gamma_{1}\right)$ and $\left(Y ; \Gamma_{2}\right)$ be abstract convex spaces. For their product, as in the Lemma 3.5 we can define $\Gamma_{X \times Y}(A):=\Gamma_{1}\left(\pi_{1}(A)\right) \times \Gamma_{2}\left(\pi_{2}(A)\right)$ for $A \in\langle X \times Y\rangle$.

Theorem 5.1. Let $(E ; \Gamma):=\left(X \times Y ; \Gamma_{X \times Y}\right)$ be the product abstract convex space, and let $f, s, t, g$ : $X \times Y \rightarrow \overline{\mathbb{R}}$ be four functions, then

$$
\mu:=\inf _{y \in Y} \sup _{x \in X} f(x, y), \quad v:=\operatorname{supinf}_{x \in X} g(x, y)
$$

Suppose that

(4.1) $f(x, y) \leq s(x, y) \leq t(x, y) \leq g(x, y)$ for each $(x, y) \in X \times Y$,

(4.2) for each $r<\mu$ and $y \in Y,\{x \in X \mid s(x, y)>r\}$ is $\Gamma_{1}$-convex; for each $r>v$ and $x \in X$, $\{y \in Y \mid t(x, y)<r\}$ is $\Gamma_{2}$-convex,

(4.3) for each $r>v$, there exists a finite set $\left\{x_{i}\right\}_{i=1}^{m} \subset X$ such that

$$
Y=\bigcup_{i=1}^{m} \operatorname{Int}\left\{y \in Y \mid f\left(x_{i}, y\right)>r\right\}
$$

(4.4) for each $r<\mu$, there exists a finite set $\left\{y_{j}\right\}_{j=1}^{n} \subset Y$ such that

$$
X=\bigcup_{j=1}^{n} \operatorname{Int}\left\{x \in X \mid g\left(x, y_{j}\right)<r\right\}
$$

If $(E ; \Gamma)$ satisfies the partial KKM principle, then

$$
\mu=\inf _{y \in Y} \sup _{x \in X} f(x, y) \leq \operatorname{supinf}_{x \in X} g(x, y)=v .
$$

Proof. Suppose that there exists a real $c$ such that

$$
\mathcal{v}=\operatorname{supinf}_{x \in X} g(x, y)<c<\inf _{y \in Y} \sup _{x \in X} f(x, y)=\mu .
$$

For the abstract convex space

$$
(E, D ; \Gamma):=\left(X \times Y,\left\{\left(x_{i}, y_{j}\right)\right\}_{i, j} ; \Gamma_{X \times Y}\right),
$$


define two maps $S: E \multimap D, T: E \multimap E$ by

$$
\begin{aligned}
S^{-}\left(x_{i}, y_{j}\right) & :=\operatorname{Int}\left\{x \in X \mid g\left(x, y_{j}\right)<c\right\} \times \operatorname{Int}\left\{y \in Y \mid f\left(x_{i}, y\right)>c\right\}, \\
T(x, y) & :=\{\bar{x} \in X \mid s(\bar{x}, y)>c\} \times\{\bar{y} \in Y \mid t(x, \bar{y})<c\},
\end{aligned}
$$

for $\left(x_{i}, y_{j}\right) \in D$ and $(x, y) \in E$, respectively. Then each $T(x, y)$ is nonempty and $\Gamma$-convex and $E$ is covered by a finite number of open sets $S^{-}\left(x_{i}, y_{j}\right)^{\prime}$ s. Moreover,

$$
\begin{aligned}
S(x, y) & \subset\left\{\left(x_{i}, y_{j}\right) \mid g\left(x, y_{j}\right)<c, f\left(x_{i}, y\right)>c\right\} \\
& \subset\{(\bar{x}, \bar{y}) \mid s(\bar{x}, y)>c, t(x, \bar{y})<c\} \subset T(x, y) .
\end{aligned}
$$

This implies that $\operatorname{co}_{\Gamma} S(x, y) \subset T(x, y)$ for all $(x, y) \in E$. Then $T$ is a $\Phi$-map. Therefore, by Theorem 3.4, we have $\left(x_{0}, y_{0}\right) \in X \times Y$ such that $\left(x_{0}, y_{0}\right) \in T\left(x_{0}, y_{0}\right)$. Therefore, $c<s\left(x_{0}, y_{0}\right) \leq$ $t\left(x_{0}, y_{0}\right)<c$, a contradiction.

Example 5.2. For convex spaces $X, Y$, and $f=s=t=g$, Theorem 5.1 reduces to that by Cho et al. [50, Theorem 8].

Corollary 5.3. Let $\left(X ; \Gamma_{1}\right)$ and $\left(Y ; \Gamma_{2}\right)$ be compact abstract convex spaces, let $(E ; \Gamma):=\left(X \times Y ; \Gamma_{X \times Y}\right)$ be the product abstract convex space, and let $f, g: X \times Y \rightarrow \overline{\mathbb{R}}$ be functions satisfying the following:

(1) $f(x, y) \leq s(x, y) \leq t(x, y) \leq g(x, y)$ for each $(x, y) \in X \times Y$,

(2) for each $x \in X, f(x, \cdot)$ is l.s.c. and $t(x, \cdot)$ is quasiconvex on $Y$,

(3) for each $y \in Y, s(\cdot, y)$ is quasiconcave and $g(\cdot, y)$ is u.s.c. on $X$.

If $(E ; \Gamma)$ satisfies the partial KKM principle, then

$$
\operatorname{minsup}_{y \in Y} f(x, y) \leq \operatorname{maxinf}_{x \in X} \sin _{y \in Y} g(x, y)
$$

Proof. Note that $y \mapsto \sup _{x \in X} f(x, y)$ is l.s.c. on $Y$ and $x \mapsto \inf _{y \in Y} g(x, y)$ is u.s.c. on $X$. Therefore, both sides of the inequality exist. Then all the requirements of Theorem 5.1 are satisfied.

Example 5.4. (1) Particular or slightly different versions of Corollary 5.3 are obtained by Liu [51], Granas [13, Théorèmes 3.1 et 3.2], and Shih and Tan [52, Theorem 4] for convex subsets of t.v.s.

(2) For $f=s, g=t$, Corollary 5.3 reduces to [27, Theorem 3].

For the case $f=s=t=g$, Corollary 5.3 reduces to the following.

Corollary 5.5 (see [27]). Let $\left(X ; \Gamma_{1}\right)$ and $\left(Y ; \Gamma_{2}\right)$ be compact abstract convex spaces and let $f$ : $X \times Y \rightarrow \overline{\mathbb{R}}$ be an extended real function such that

(1) for each $x \in X, f(x, \cdot)$ is l.s.c. and quasiconvex on $Y$,

(2) for each $y \in Y, f(\cdot, y)$ is u.s.c. and quasiconcave on $X$. 
If $\left(X \times Y ; \Gamma_{X \times Y}\right)$ satisfies the partial KKM principle, then

(i) $f$ has a saddle point $\left(x_{0}, y_{0}\right) \in X \times Y$,

(ii) one has

$$
\max _{x \in X} \min _{y \in Y} f(x, y)=\min _{y \in Y} \max _{x \in X} f(x, y) .
$$

Example 5.6. We list historically well-known particular forms of Corollary 5.5 in chronological order as follows.

(1) von Neumann [1], Kakutani [3]. $X$ and $Y$ are compact convex subsets of Euclidean spaces and $f$ is continuous.

(2) Nikaidô [53]. Euclidean spaces above are replaced by Hausdorff topological vector spaces, and $f$ is continuous in each variable.

(3) Sion [54]. $X$ and $Y$ are compact convex subsets of topological vector spaces in Corollary 5.5.

(4) Komiya [55, Theorem 3]. $X$ and $Y$ are compact convex spaces in the sense of Komiya.

(5) Horvath [36, Proposition 5.2]. $X$ and $Y$ are $c$-spaces with $Y$ being compact and without assuming the compactness of $X$.

In these two examples, Hausdorffness of $Y$ is assumed since they used the partition of unity argument.

(6) Bielawski [29, Theorem (4.13)]. $X$ and $Y$ are compact spaces having certain simplicial convexities.

(7) Park [17, Theorem 5]. $X$ and $Y$ are G-convex spaces.

In 1999, we deduced the following von Neumann-Sion type minimax theorem for Gconvex spaces based on a continuous selection theorem:

Theorem 5.7 (see [17]). Let $\left(X, \Gamma_{1}\right)$ and $\left(Y, \Gamma_{2}\right)$ be G-convex spaces, $Y$ Hausdorff compact, $f$ : $X \times Y \rightarrow \bar{R}$ an extended real function, and $\mu:=\sup _{x \in X} \inf _{y \in Y} f(x, y)$. Suppose that

(5.1) $f(x, \cdot)$ is l.s.c. on $Y$ and $\{y \in Y \mid f(x, y)<r\}$ is $\Gamma_{2}$-convex for each $x \in X$ and $r>\mu$, Then

(5.2) $f(\cdot, y)$ is u.s.c. on $X$ and $\{x \in X \mid f(x, y)>r\}$ is $\Gamma_{1}$-convex for each $y \in Y$ and $r>\mu$.

$$
\sup _{x \in X} \min _{y \in Y} f(x, y)=\min _{y \in Y} \sup _{x \in X} f(x, y)
$$

Example 5.8. (1) Komiya [55, Theorem 3]. $X$ and $Y$ are compact convex spaces in the sense of Komiya.

(2) Slightly different form of Theorem 5.7 can be seen in [17] with different proof.

\section{Collective Fixed Point Theorems}

We have the following collective fixed point theorem.

Theorem 6.1. Let $\left\{\left(X_{i} ; \Gamma_{i}\right)\right\}_{i=1}^{n}$ be a finite family of compact abstract convex spaces such that $(X ; \Gamma)=$ $\left(\prod_{i=1}^{n} X_{i} ; \Gamma\right)$ satisfies the partial KKM principle, and for each $i, T_{i}: X \multimap X_{i}$ is a $\Phi$-map. Then there 
exists a point $x \in X$ such that $x \in T(x):=\prod_{i=1}^{n} T_{i}(x)$, that is, $x_{i}=\pi_{i}(x) \in T_{i}(x)$ for each $i=1,2, \ldots, n$.

Proof. Let $S_{i}: X \multimap X_{i}$ be the companion map corresponding to the $\Phi$-map $T_{i}$. Define $S: X \multimap$ $X$ by

$$
S(x):=\prod_{i=1}^{n} S_{i}(x) \quad \text { for each } x \in X
$$

We show that $T$ is a $\Phi$-map with the companion map $S$. In fact, we have

$$
x \in S^{-}(y) \Longleftrightarrow y \in S(x) \Longleftrightarrow y_{i} \in S_{i}(x) \quad \text { for each } i \Longleftrightarrow x \in S_{i}^{-}\left(y_{i}\right) \quad \text { for each } i,
$$

where $y=\left\{y_{1}, \ldots, y_{n}\right\}$. Since each $S_{i}^{-}\left(y_{i}\right)$ is open, we have

(a) for each $y \in X, S^{-}(y)=\bigcap_{i=1}^{n} S_{i}^{-}\left(y_{i}\right)$ is open.

Note that

$$
M \in\langle S(x)\rangle \Longrightarrow \pi_{i}(M) \in\left\langle S_{i}(x)\right\rangle \Longrightarrow \Gamma_{i}\left(\pi_{i}(M)\right) \subset T_{i}(x),
$$

and hence,

$$
\Gamma_{M}=\prod_{i=1}^{n} \Gamma_{i}\left(\pi_{i}(M)\right) \subset \prod_{i=1}^{n} T_{i}(x)=T(x) .
$$

Therefore, we have

(b) for each $x \in X, M \in\langle S(x)\rangle$ implies that $\Gamma_{M} \subset T(x)$.

Moreover, let $x \in X$. Since $S_{i}: X \multimap X_{i}$ is the companion map corresponding to the $\Phi$-map $T_{i}$, for each $i$, there exists $j=j(i)$ such that

$$
x \in S_{i}^{-}\left(y_{i, j}\right) \Rightarrow y_{i, j} \in S_{i}(x) \Rightarrow y \in \prod_{i=1}^{n} S_{i}(x)=S(x) \Rightarrow x \in S^{-}(y),
$$

where $y:=\left(y_{1, j(1)}, \ldots, y_{n, j(n)}\right)$. Since $X$ is compact, we have

(c) $X=\bigcup_{z \in M} S^{-}(z)$ for some $M \in\langle X\rangle$. fixed point.

Since $(X ; \Gamma)$ satisfies the partial KKM principle, by Theorem 3.4, the $\Phi$-map $T$ has a

Example 6.2. (1) If $n=1, X$ is a convex space, and $S=T$, then Theorem 6.1 reduces to the well-known Fan-Browder fixed point theorem; see Park [56].

(2) For the case $n=1$, Theorem 6.1 for a convex space $X$ was obtained by Ben-ElMechaiekh et al. [69, Theorem 1] and Simons [57, Theorem 4.3]. This was extended by many authors; see Park [56]. 
We have already the following collective fixed point theorem for arbitrary family of G-convex spaces.

Theorem 6.3 (see [40]). Let $\left\{\left(X_{i} ; \Gamma_{i}\right)\right\}_{i \in I}$ be a family of compact Hausdorff $G$-convex spaces, $X=$ $\prod_{i \in I} X_{i}$, and for each $i \in I$, let $T_{i}: X \multimap X_{i}$ be a $\Phi$-map. Then there exists a point $x \in X$ such that $x \in T(x):=\prod_{i \in I} T_{i}(x)$, that is, $x_{i}=\pi_{i}(x) \in T_{i}(x)$ for each $i \in I$.

Example 6.4. In case when $\left(X_{i} ; \Gamma_{i}\right)$ are all $H$-spaces, Theorem 6.3 reduces to Tarafdar [58, Theorem 2.3]. This is applied to sets with $H$-convex sections [58, Theorem 3.1] and to existence of equilibrium point of an abstract economy [58, Theorem 4.1 and Corollary 4.1]. These results also can be extended to G-convex spaces and we will not repeat then here.

Remark 6.5. Each of Theorems 6.1, 7.1, 8.1,9.1, and 9.4, respectively, in this paper is based on the KKM method and concerns with finite families of abstract convex spaces such that their product satisfies the partial KKM principle. Each of them has a corresponding Theorems 6.3, 7.3, 8.3, 9.2 and 9.6, respectively, based on continuous selection method for infinite families of Hausdorff G-convex spaces. Note that for finite families the Hausdorffness is redundant in these corresponding theorems.

\section{Intersection Theorems for Sets with Convex Sections}

In our previous work [17], from a G-convex space version of the Fan-Browder fixed point theorem, we deduced a Fan-type intersection theorem for $n$ subsets of a cartesian product of $n$ compact $G$-convex spaces. This was applied to obtain a von Neumann-sion-type minimax theorem and a Nash-type equilibrium theorem for G-convex spaces.

In the present section, we generalize the abovementioned intersection theorem to product abstract convex spaces satisfying the partial KKM principle.

The collective fixed point theorem in Section 6 can be reformulated to a generalization of various Fan-type intersection theorems for sets with convex sections as follows.

Let $\left\{X_{i}\right\}_{i \in I}$ be a family of sets, and let $i \in I$ be fixed. Let

$$
X=\prod_{j \in I} X_{j}, \quad X^{i}=\prod_{j \in I \backslash\{i\}} X_{j} .
$$

If $x^{i} \in X^{i}$ and $j \in I \backslash\{i\}$, then let $x_{j}^{i}$ denote the $j$ th coordinate of $x^{i}$. If $x^{i} \in X^{i}$ and $x_{i} \in X_{i}$, then let $\left[x^{i}, x_{i}\right] \in X$ be defined as follows: its $i$ th coordinate is $x_{i}$ and for $j \neq i$ the $j$ th coordinate is $x_{j}^{i}$. Therefore, any $x \in X$ can be expressed as $x=\left[x^{i}, x_{i}\right]$ for any $i \in I$, where $x^{i}$ denotes the projection of $x$ in $X^{i}$.

Theorem 7.1. Let $\left\{\left(X_{i} ; \Gamma_{i}\right)\right\}_{i=1}^{n}$ be a finite family of compact abstract convex spaces such that $(X ; \Gamma)=$ $\left(\prod_{i=1}^{n} X_{i} ; \Gamma\right)$ satisfies the partial KKM principle and, for each $i$, let $A_{i}$ and $B_{i}$ be subsets of $X$ satisfying the following.

(7.1) For each $x^{i} \in X^{i}, \emptyset \neq \operatorname{co}_{\Gamma_{i}} B_{i}\left(x^{i}\right) \subset A_{i}\left(x^{i}\right):=\left\{y_{i} \in X_{i} \mid\left[x^{i}, y_{i}\right] \in A_{i}\right\}$.

(7.2) For each $y_{i} \in X_{i}, B_{i}\left(y_{i}\right):=\left\{x^{i} \in X^{i} \mid\left[x^{i}, y_{i}\right] \in B_{i}\right\}$ is open in $X^{i}$.

Then $\bigcap_{i=1}^{n} A_{i} \neq \emptyset$.

Proof. We apply Theorem 6.1 with multimaps $S_{i}, T_{i}: X \multimap X_{i}$ given by $S_{i}(x):=B_{i}\left(x^{i}\right)$ and $T_{i}(x):=A_{i}\left(x^{i}\right)$ for each $x \in X$. Then for each $i$ we have the following. 
(a) For each $x \in X$, we have $\emptyset \neq \operatorname{co}_{\Gamma_{i}} S_{i}(x) \subset T_{i}(x)$.

(b) For each $y_{i} \in X_{i}$, we have

$$
x \in S_{i}^{-}\left(y_{i}\right) \Longleftrightarrow y_{i} \in S_{i}(x)=B_{i}\left(x^{i}\right) \Longleftrightarrow\left[x^{i}, y_{i}\right] \in B_{i} \subset X^{i} \times X_{i}=X
$$

Hence,

$$
S_{i}^{-}\left(y_{i}\right)=\left\{x=\left[x^{i}, x_{i}\right] \in X \mid x^{i} \in B_{i}\left(y_{i}\right), x_{i} \in X_{i}\right\}=B_{i}\left(y_{i}\right) \times X_{i}
$$

Note that $S_{i}^{-}\left(y_{i}\right)$ is open in $X=X^{i} \times X_{i}$ and that $T_{i}$ is a $\Phi$-map. Therefore, by Theorem 6.1, there exists $\widehat{x} \in X$ such that $\widehat{x}_{i} \in T_{i}(\widehat{x})=A_{i}\left(\widehat{x}^{i}\right)$ for all $i$. Hence $\widehat{x}=\left[\widehat{x}^{i}, \widehat{x}_{i}\right] \in \bigcap_{i=1}^{n} A_{i} \neq \emptyset$.

Example 7.2. For convex spaces $X_{i}$, particular forms of Theorem 7.1 have appeared as follows:

(1) Fan [10, Théeorème 1]. $A_{i}=B_{i}$ for all $i$.

(2) Fan [11, Theorem $\left.1^{\prime}\right] . n=2$ and $A_{i}=B_{i}$ for $i=1,2$.

From these results, Fan [11] deduced an analytic formulation, fixed point theorems, extension theorems of monotone sets, and extension theorems for invariant vector subspaces.

For particular types of G-convex spaces, Theorem 7.1 was known as follows.

(3) Bielawski [29, Proposition (4.12) and Theorem (4.15)]. $X_{i}$ have the finitely local convexity.

(4) Kirk et al. [32, Theorem 5.2]. $X_{i}$ are hyperconvex metric spaces.

(5) Park [17, Theorem 4], [18, Theorem 19]. In [17], from a G-convex space version of the Fan-Browder fixed point theorem, we deduced a Fan-type intersection theorem for $n$ subsets of a cartesian product of $n$ compact $G$-convex spaces. This was applied to obtain a von Neumann-Sion-type minimax theorem and a Nash-type equilibrium theorem for $G$-convex spaces.

(6) Park [27, Theorem 4]. We gave a different proof.

In [22], a collective fixed point theorem was reformulated to a generalization of various Fan-type intersection theorems for arbitrary number of sets with convex sections as follows.

Theorem 7.3 (see [22]). Let $\left\{\left(X_{i} ; \Gamma_{i}\right)\right\}_{i \in I}$ be a family of Hausdorff compact G-convex spaces and, for each $i \in I$, let $A_{i}$ and $B_{i}$ be subsets of $X=\prod_{i \in I} X_{i}$ satisfying the following.

(7.1)' For each $x^{i} \in X^{i}, \emptyset \neq \operatorname{co}_{\Gamma_{i}} B_{i}\left(x^{i}\right) \subset A_{i}\left(x^{i}\right):=\left\{y_{i} \in X_{i} \mid\left[x^{i}, y_{i}\right] \in A_{i}\right\} .(7.2)^{\prime}$ For each $y_{i} \in X_{i}, B_{i}\left(y_{i}\right):=\left\{x^{i} \in X^{i} \mid\left[x^{i}, y_{i}\right] \in B_{i}\right\}$ is open in $X^{i}$.

Then $\bigcap_{i \in I} A_{i} \neq \emptyset$.

Example 7.4. For convex subsets $X_{i}$ of topological vector spaces, particular forms of Theorem 7.3 have appeared as follows.

(1) Ma [12, Theorem 2]. The case $A_{i}=B_{i}$ for all $i \in I$ with a different proof is given.

(2) Chang [59, Theorem 4.2] obtained Theorem 7.3 with a different proof. The author also obtained a noncompact version of Theorem 7.3 as in [59, Theorem 4.3].

(3) Park [19, Theorem 4.2]. $X_{i}$ are convex spaces.

Note that if $I$ is finite in Theorem 7.3, the Hausdorffness is redundant by Theorem 7.1. 


\section{The Fan-Type Analytic Alternatives}

From the intersection Theorem 7.1, we can deduce the following equivalent form of a generalized Fan-type minimax inequality or analytic alternative. Our method is based on that of Fan $[9,10]$ and Ma [12].

Theorem 8.1. Let $\left\{\left(X_{i} ; \Gamma_{i}\right)\right\}_{i=1}^{n}$ be a finite family of compact abstract convex spaces such that $(X ; \Gamma)=$ $\left(\prod_{i=1}^{n} X_{i} ; \Gamma\right)$ satisfies the partial KKM principle and, for each $i$, let $f_{i}, g_{i}: X=X^{i} \times X_{i} \rightarrow \mathbb{R}$ be real functions satisfying

(8.1) $f_{i}(x) \leq g_{i}(x)$ for each $x \in X$,

(8.2) for each $x^{i} \in X^{i}, x_{i} \mapsto g_{i}\left[x^{i}, x_{i}\right]$ is quasiconcave on $X_{i}$,

(8.3) for each $x_{i} \in X_{i}, x^{i} \mapsto f_{i}\left[x^{i}, x_{i}\right]$ is l.s.c. on $X^{i}$.

Let $\left\{t_{i}\right\}_{i=1}^{n}$ be a family of real numbers. Then either

(a) there exist an $i$ and an $x^{i} \in X^{i}$ such that

$$
f_{i}\left[x^{i}, y_{i}\right] \leq t_{i} \quad \forall y_{i} \in X_{i}
$$

or (b) there exists an $x \in X$ such that

$$
g_{i}(x)>t_{i} \quad \forall i=1,2, \ldots, n
$$

Proof. Suppose that (a) does not hold, that is, for any $i$ and any $x^{i} \in X^{i}$, there exists an $x_{i} \in X_{i}$ such that $f_{i}\left[x^{i}, x_{i}\right]>t_{i}$. Let

$$
A_{i}:=\left\{x \in X \mid g_{i}(x)>t_{i}\right\}, \quad B_{i}=\left\{x \in X \mid f_{i}(x)>t_{i}\right\}
$$

for each $i$. Then

(1) for each $x^{i} \in X^{i}, \emptyset \neq B_{i}\left(x^{i}\right) \subset A_{i}\left(x^{i}\right)$,

(2) for each $x^{i} \in X^{i}, A_{i}\left(x^{i}\right)$ is $\Gamma_{i}$-convex,

(3) for each $y_{i} \in X_{i}, B_{i}\left(y_{i}\right)$ is open in $X^{i}$.

Therefore, by Theorem 7.1, there exists an $x \in \bigcap_{i=1}^{n} A_{i}$. This is equivalent to (b).

Example 8.2. Fan [9, Théorème 2], [10, Theorem 3]. $X_{i}$ are convex subsets of t.v.s., and $f_{i}=g_{i}$ for all $i$. From this, fan $[9,10]$ deduced Sion's minimax theorem [54], the Tychonoff fixed point theorem, solutions to systems of convex inequalities, extremum problems for matrices, and a theorem of Hardy-Littlewood-Pólya.

From the intersection Theorem 7.3, we can deduce the following equivalent form of a generalized Fan-type minimax inequality or analytic alternative. 
Theorem 8.3 (see [22]). Let $\left\{\left(X_{i} ; \Gamma_{i}\right)\right\}_{i \in I}$ be a family of compact Hausdorff G-convex spaces and, for each $i \in I$, let $f_{i}, g_{i}: X=X^{i} \times X_{i} \rightarrow \mathbb{R}$ be real functions as in Theorem 8.1. Then the conclusion of Theorem 8.1 holds.

Example 8.4. (1) Ma [12, Theorem 3]. $X_{i}$ are convex subsets of t.v.s. and $f_{i}=g_{i}$ for all $i \in I$.

(2) Park [19, Theorem 8.1]. $X_{i}$ are convex spaces.

Remark 8.5. (1) We obtained Theorem 8.1 from Theorem 7.1. As was pointed out by Fan [9] for his case, we can deduce Theorem 7.1 from Theorem 8.1 by considering the characteristic functions of the sets $A_{i}$ and $B_{i}$.

(2) The conclusion of Theorems 8.1 and 8.3 can be stated as follows

$$
\operatorname{minsup}_{x^{i} \in X^{i}} f_{x_{i} \in X_{i}}\left[x^{i}, x_{i}\right]>t_{i} \quad \forall i
$$

then (b) holds; see Fan $[9,10]$.

(3) For $I=\{1,2\}$, Theorems 8.1 and 8.3 imply the Fan minimax inequality.

\section{The Nash-Type Equilibrium Theorems}

From Theorem 8.1, we obtain the following form of the Nash-Fan-type equilibrium theorems in [27] with different proofs.

Theorem 9.1. Let $\left\{\left(X_{i} ; \Gamma_{i}\right)\right\}_{i=1}^{n}$ be a finite family of compact abstract convex spaces such that $(X ; \Gamma)=$ $\left(\prod_{i=1}^{n} X_{i} ; \Gamma\right)$ satisfies the partial KKM principle and, for each $i$, let $f_{i}, g_{i}: X=X^{i} \times X_{i} \rightarrow \mathbb{R}$ be real functions such that

(9.0) $f_{i}(x) \leq g_{i}(x)$ for each $x \in X$,

(9.1) for each $x^{i} \in X^{i}, x_{i} \mapsto g_{i}\left[x^{i}, x_{i}\right]$ is quasiconcave on $X_{i}$,

(9.2) for each $x^{i} \in X^{i}, x_{i} \mapsto f_{i}\left[x^{i}, x_{i}\right]$ is u.s.c. on $X_{i}$,

(9.3) for each $x_{i} \in X_{i}, x^{i} \mapsto f_{i}\left[x^{i}, x_{i}\right]$ is l.s.c. on $X^{i}$.

Then there exists a point $\hat{x} \in X$ such that

$$
g_{i}(\widehat{x}) \geq \max _{y_{i} \in X_{i}} f_{i}\left[\widehat{x}^{i}, y_{i}\right] \quad \forall i=1,2, \ldots, n
$$

Proof. Since each $X_{i}$ is compact, by (9.2), for any $\varepsilon>0, t_{i}:=\max _{y_{i} \in X_{i}} f_{i}\left[x^{i}, y_{i}\right]-\varepsilon$ exists for all $x^{i} \in X^{i}$ and all $i$. Hence Theorem 8.1(a) does not hold. Then by Theorem 8.1(b), there exists an $\widehat{x} \in E$ such that $g_{i}(\widehat{x})>t_{i}=\max _{y_{i} \in X_{i}} f_{i}\left[\hat{x}^{i}, y_{i}\right]-\varepsilon$ for all $i \in I$. Since $\varepsilon>0$ is arbitrary, the conclusion follows.

This is not comparable to the following generalized Nash-Ma type theorem:

Theorem 9.2 (see [22]). Let $\left\{\left(X_{i} ; \Gamma_{i}\right)\right\}_{i \in I}$ be a family of compact Hausdorff G-convex spaces and, for each $i \in I$, let $f_{i}, g_{i}: X=X^{i} \times X_{i} \rightarrow \mathbb{R}$ be real functions satisfying (9.0)-(9.3). Then there exists a point $\hat{x} \in X$ such that

$$
g_{i}(\widehat{x}) \geq \max _{y_{i} \in X_{i}} f_{i}\left[\widehat{x}^{i}, y_{i}\right] \quad \forall i \in I
$$


Example 9.3. Park [19, Theorem 8.2]. $X_{i}$ are convex spaces.

From Theorem 9.1 for $f_{i}=g_{i}$, we obtain the following form of the Nash-Fan-type equilibrium theorem for abstract convex spaces.

Theorem 9.4. Let $\left\{\left(X_{i} ; \Gamma_{i}\right)\right\}_{i=1}^{n}$ be a finite family of compact abstract convex spaces such that $(X ; \Gamma)=$ $\left(\prod_{i=1}^{n} X_{i} ; \Gamma\right)$ satisfies the partial KKM principle and, for each $i \in I$, let $f_{i}: X \rightarrow \mathbb{R}$ be a function such that

(10.1) for each $x^{i} \in X^{i}, x_{i} \mapsto f_{i}\left[x^{i}, x_{i}\right]$ is quasiconcave on $X_{i}$,

(10.2) for each $x^{i} \in X^{i}, x_{i} \mapsto f_{i}\left[x^{i}, x_{i}\right]$ is u.s.c. on $X_{i}$,

(10.3) for each $x_{i} \in X_{i}, x^{i} \mapsto f_{i}\left[x^{i}, x_{i}\right]$ is l.s.c. on $X^{i}$.

Then there exists a point $\widehat{x} \in X$ such that

$$
f_{i}(\widehat{x})=\max _{y_{i} \in X_{i}} f_{i}\left[\widehat{x}^{i}, y_{i}\right] \quad \forall i=1,2, \ldots, n
$$

Example 9.5. For continuous functions $f_{i}$, a number of particular forms of Theorem 9.4 have appeared for convex subsets $X_{i}$ of Hausdorff topological vector spaces as follows:

(1) Nash [5, Theorem 1] where $X_{i}$ are subsets of Euclidean spaces,

(2) Nikaido and Isoda [60, Theorem 3.2],

(3) Fan [10, Theorem 4],

(4) Tan et al. [61, Theorem 2.1].

For particular types of $G$-convex spaces $X_{i}$ and continuous functions $f_{i}$, particular forms of Theorem 9.4 have appeared as follows.

(5) Bielawski [29, Theorem 4.16]. $X_{i}$ have the finitely local convexity.

(6) Kirk et al. [32, Theorem 5.3]. $X_{i}$ are hyperconvex metric spaces.

(7) Park [17, Theorem 6.1], [18, Theorem 20]. $X_{i}$ are G-convex spaces.

(8) Park [21, Theorem 4.7]. A variant of Theorem 9.4 is under the hypothesis that $(X ; \Gamma)$ is a compact $G$-convex space and $f_{1}, \ldots, f_{n}: X \rightarrow \mathbb{R}$ are continuous functions.

(9) González et al. [31]. Each $X_{i}$ is a compact, sequentially compact $L$-space and each $f_{i}$ is continuous as in 8.

(10) Briec and Horvath [30, Theorem 3.2]. Each $X_{i}$ is a compact $B$-convex set and each $f_{i}$ is continuous as in 8 .

From Theorem 9.2, we obtain the following generalization of the Nash-Ma-type equilibrium theorem for $G$-convex spaces.

Theorem 9.6 (see [22]). Let $\left\{\left(X_{i} ; \Gamma_{i}\right)\right\}_{i \in I}$ be a family of compact Hausdorff G-convex spaces and, for each $i \in I$, let $f_{i}: X \rightarrow \mathbb{R}$ be a function satisfying conditions (10.1)-(10.3). Then there exists a point $\hat{x} \in X$ such that

$$
f_{i}(\widehat{x})=\max _{y_{i} \in X_{i}} f_{i}\left[\widehat{x}^{i}, y_{i}\right] \quad \forall i \in I
$$


Example 9.7. For continuous functions $f_{i}$ and for convex subsets $X_{i}$ of Hausdorff topological vector spaces, Theorem 9.6 was due to Ma [12, Theorem 4].

The point $\widehat{x}$ in the conclusion of Theorems 9.4 or 9.6 is called a Nash equilibrium. This concept is a natural extension of the local maxima and the saddle point as follows.

In case $I$ is a singleton, we obtain the following.

Corollary 9.8. Let $X$ be a closed bounded convex subset of a reflexive Banach space $E$ and $f: X \rightarrow \mathbb{R}$ a quasiconcave u.s.c. function. Then $f$ attains its maximum on $X$, that is, there exists an $\hat{x} \in X$ such that $f(\widehat{x}) \geq f(x)$ for all $x \in X$.

Proof. Let $E$ be equipped with the weak topology. Then, by the Hahn-Banach theorem, $f$ is still u.s.c. because $f$ is quasiconcave, and $X$ is still closed. Being bounded, $X$ is contained in some closed ball which is weakly compact. Since any closed subset of a compact set is compact, $X$ is (weakly) compact. Now, by Theorem 9.4 for a single family, we have the conclusion.

Corollary 9.8 is due to Mazur and Schauder in 1936. Some generalized forms of Corollary 9.8 were known by Park et al. $[62,63]$.

For $I=\{1,2\}$, Theorem 9.4 reduces to Corollary 5.5 as follows.

Proof of Corollary 5.5 from Theorem 9.4. Let $f_{1}(x, y):=-f(x, y)$ and $f_{2}(x, y):=f(x, y)$. Then all of the requirements of Theorem 9.4 are satisfied. Therefore, by Theorem 9.4, there exists a point $\left(x_{0}, y_{0}\right) \in X \times Y$ such that

$$
f_{1}\left(x_{0}, y_{0}\right)=\max _{y \in Y} f_{1}\left(x_{0}, y\right), \quad f_{2}\left(x_{0}, y_{0}\right)=\max _{x \in X} f_{2}\left(x, y_{0}\right)
$$

Therefore, we have

$$
\begin{gathered}
-f\left(x_{0}, y_{0}\right)=f_{1}\left(x_{0}, y_{0}\right) \geq f_{1}\left(x_{0}, y\right)=-f\left(x_{0}, y\right) \quad \forall y \in Y, \\
f\left(x_{0}, y_{0}\right)=f_{2}\left(x_{0}, y_{0}\right) \geq f_{2}\left(x, y_{0}\right)=f\left(x, y_{0}\right) \quad \forall x \in X .
\end{gathered}
$$

Hence

$$
f\left(x, y_{0}\right) \leq f\left(x_{0}, y_{0}\right) \leq f\left(x_{0}, y\right) \quad \forall(x, y) \in X \times Y .
$$

Therefore

$$
\max _{x \in X} f\left(x, y_{0}\right) \leq f\left(x_{0}, y_{0}\right) \leq \min _{y \in X} f\left(x_{0}, y\right)
$$

This implies that

$$
\min _{y \in X} \max _{x \in X} f(x, y) \leq f\left(x_{0}, y_{0}\right) \leq \max _{x \in X} \min _{y \in X} f(x, y) .
$$


On the other hand, we have trivially

$$
\min _{y \in X} f(x, y) \leq \max _{x \in X} f(x, y)
$$

and hence

$$
\max _{x \in X} \min _{y \in X} f(x, y) \leq \min _{y \in X} \max _{x \in X} f(x, y) .
$$

Therefore, we have the conclusion.

\section{Historical Remarks on Related Results}

(I) As we have seen in Sections 1-3, we have three methods in our subject as follows:

(1) fixed point method-applications of the Kakutani theorem and its various generalizations (e.g., acyclic-valued multimaps, admissible maps, or better admissible maps in the sense of Park); see [3-8, 10, 12, 14-16, 19, 20, 23, 28, 42, 53, 64-68] and others,

(2) continuous selection method-applications of the fact that Fan-Browder-type maps have continuous selections under certain assumptions like Hausdorffness and compactness of relevant spaces; see [17, 22, 36, 39, 40, 58, 69, 70] and others,

(3) the KKM method-as for the Sion theorem, direct applications of the KKM theorem, or its equivalents like the Fan-Browder fixed point theorem for which we do not need the Hausdorffness; see $[9,11,17,21,24-27,30,31,33,35-37,39,43,45,47-$ $50,54,55,57,59,71,72]$ and others.

For Case (1), we will study it elsewhere and, in this paper, we are mainly concerned with Cases (2) and (3).

(II) An upper semicontinuous (u.s.c.) multimap with nonempty compact convex values is called a Kakutani map. The Fan-Glicksberg theorem was extended by Himmelberg [8] in 1972 for compact Kakutani maps instead of assuming compactness of domains. In 1990, Lassonde [67] extended the Himmelberg theorem to multimaps factorizable by Kakutani maps through convex sets in Hausdorff topological vector spaces. Moreover, Lassonde applied his theorem to game theory and obtained a von Neumann-type intersection theorem for finite number of sets and a Nash-type equilibrium theorem comparable to Debreu's social equilibrium existence theorem [66].

Fixed point theorems extending the Kakutani theorem can be applied to particular forms of results in this paper. Since such extended theorems usually assume Hausdorffness and certain (abstract) local convexity of the related space, their applicability is restrictive.

(III) In 1946, the Kakutani theorem was extended for acyclic maps by Eilenberg and Montgomery [73]. This result was applied by Debreu [66] to the social equilibrium existence theorem and related results.

(IV) Since 1996 [72], many authors have published some results of the present paper for hyperconvex metric spaces. For example, Kirk et al. in 2000 [32] established the KKM theorem, its equivalent formulations, fixed point theorems, and the Nash theorem for hyperconvex metric spaces. However, already in 1993, Horvath [37] found that hyperconvex metric spaces are a particular type of $c$-spaces. 
(V) In 1998 [16], an acyclic version of the social equilibrium existence theorem of Debreu is obtained. This is applied to deduce acyclic versions of theorems on saddle points, minimax theorems, and the following Nash equilibrium theorem.

Corollary 10.1 (see [16]). Let $\left\{X_{i}\right\}_{i=1}^{n}$ be a family of acyclic polyhedra, $X=\prod_{i=1}^{n} X_{i}$, and for each $i$, $f_{i}: X \rightarrow \overline{\mathbb{R}}$ a continuous function such that

(0) for each $x^{i} \in X^{i}$ and each $\alpha \in \overline{\mathbb{R}}$, the set

$$
\left\{x_{i} \in X_{i} \mid f_{i}\left(x^{i}, x_{i}\right) \geq \alpha\right\}
$$

is empty or acyclic.

Then there exists a point $\hat{a} \in X$ such that

$$
f_{i}(\widehat{a})=\max _{y_{i} \in X_{i}} f_{i}\left(\widehat{a}^{i}, y_{i}\right) \quad \forall i \in I \text {. }
$$

(VI) In the present paper, for abstract convex spaces, we notice that the partial KKM principle $\Rightarrow$ the Fan-Browder fixed point theorem $\Rightarrow$ the Nash equilibrium theorem, with or without additional intermediate steps. This procedure can be called "from the KKM principle to the Nash equilibria" (simply, "K to N"); see [27].

In 1999 [17], we obtained a "K to N" for G-convex spaces. These results extended and unified a number of known results for particular types of G-convex spaces; see also [1821, 42]. Therefore, the procedure also holds for Lassonde type-convex spaces, Horvath's cspace, hyperconvex metric spaces, and others.

(VII) In 2000 [20] and 2002 [23], we applied our fixed point theorem for compact compositions of acyclic maps on admissible (in the sense of Klee) convex subsets of a t.v.s. to obtain a cyclic coincidence theorem for acyclic maps, generalized von Neumann-type intersection theorems, the Nash type equilibrium theorems, and the von Neumann minimax theorem.

The following examples are generalized forms of quasi equilibrium theorem or social equilibrium existence theorems which directly imply generalizations of the Nash-Ma-type equilibrium existence theorem.

Theorem 10.2 (see [20]). Let $\left\{X_{i}\right\}_{i=1}^{n}$ be a family of convex sets, each in a t.v.s. $E_{i}, K_{i}$ a nonempty compact subset of $X_{i}, S_{i}: X \multimap K_{i}$ a closed map, and $f_{i}, g_{i}: X=X^{i} \times X_{i} \rightarrow \mathbb{R}$ u.s.c. functions for each $i$.

Suppose that, for each $i$,

(i) $g_{i}(x) \leq f_{i}(x)$ for each $x \in X$,

(ii) the function $M_{i}$ defined on $X$ by

$$
M_{i}(x)=\max _{y \in S_{i}(x)} g_{i}\left(x^{i}, y\right) \text { for } x \in X
$$

is l.s.c., and 
Fixed Point Theory and Applications

(iii) for each $x \in X$, the set

$$
\left\{y \in S_{i}(x) \mid f_{i}\left(x^{i}, y\right) \geq M_{i}(x)\right\}
$$

is acyclic.

If $X$ is admissible in $E=\prod_{j=1}^{n} E_{j}$, then there exists an $\hat{x} \in K$ such that, for each $i$,

$$
\widehat{x}_{i} \in S_{i}(\widehat{x}), \quad f_{i}\left(\widehat{x}^{i}, \widehat{x}_{i}\right) \geq g_{i}\left(\widehat{x}^{i}, y\right) \quad \forall y \in S_{i}(\widehat{x})
$$

Theorem 10.3 (see [23]). Let $X_{0}$ be a topological space and let $\left\{X_{i}\right\}_{i=1}^{n}$ be a family of convex sets, each in a t.v.s. $E_{i}$. For each $i=0,1, \ldots, n$, let $S_{i}: X^{i} \multimap X_{i}$ be a closed map with compact values, and $f_{i}, g_{i}: X=\prod_{i=0}^{n} X_{i} \rightarrow R$ u.s.c. real-valued functions.

Suppose that, for each $i$,

(i) $g_{i}(x) \leq f_{i}(x)$ for each $x \in X$,

(ii) the function $M_{i}: X^{i} \rightarrow R$ defined by

$$
M_{i}\left(x^{i}\right)=\max _{y_{i} \in S_{i}\left(x^{i}\right)} g_{i}\left(x^{i}, y_{i}\right) \quad \text { for } x^{i} \in X^{i}
$$

is l.s.c., and

(iii) for each $x^{i} \in X^{i}$, the set

$$
\left\{x_{i} \in S_{i}\left(x^{i}\right) \mid f_{i}\left(x^{i}, x_{i}\right) \geq M_{i}\left(x^{i}\right)\right\}
$$

is acyclic.

If $X^{0}$ is admissible in $E^{0}=\prod_{j=1}^{n} E_{j}$ and if all the maps $S_{i}$ are compact except possibly $S_{n}$ and $S_{n}$ is u.s.c., then there exists an equilibrium point $\widehat{x} \in X$, that is,

$$
\widehat{x}_{i} \in S_{i}\left(\widehat{x}^{i}\right), \quad f_{i}(\widehat{x}) \geq \max _{y_{i} \in S_{i}\left(x^{i}\right)} g_{i}\left(\widehat{x}^{i}, y\right) \quad \forall i \in Z_{n+1} .
$$

The following are particular results of ours for G-convex spaces or KKM spaces.

(VIII) In 2001 [22], we obtained generalized forms of the von Neumann-Sion-type minimax theorem, the Fan-Ma intersection theorem, the Fan-Ma type analytic alternative, and the Nash-Ma-equilibrium theorem for G-convex spaces. In [22], all G-convex spaces were assumed to be Hausdorff because the results are based on a selection theorem in [40], where Hausdorffness was indispensable.

As we have seen already in Theorems 7.3, 8.3, 9.2, and 9.6, results following the selection theorem in [40] hold for arbitrary family of Hausdorff $G$-convex spaces. But corresponding results following the partial KKM principle hold for finite families of abstract convex spaces. At present, we have no way to unify these three cases, namely, (1) fixed point method, (2) selection method, and (3) KKM method. 
(IX) In 2001, for any topological semilattice $(X, \leq)$ with path-connected interval introduced by Horvath and Llinares [39], the KKM theorem, the Fan-Browder theorem, and the Nash theorem are shown by Luo [33]. Note that such semilattice is known to be a Gconvex space.

(X) Cain and González [71] considered relationship among some subclasses of the class of G-convex spaces and introduced a subclass of the so-called $L$-spaces. In 2007, González et al. [31] repeated to show that $G$-convex spaces and $L$-spaces satisfy the partial KKM principle. They added that $L$-spaces satisfy the properties of the Fan type minimax inequality, Fan-Browder-type fixed point, and the Nash-type equilibrium. All of such results are already known for more general $G$-convex spaces.

(XI) In 2008, Kulpa and Szymanski [45] introduced a series of theorems called Infimum Principles in simplicial spaces. As for applications, they derive fixed point theorems due to Schauder, Tychnoff, Kakutani, and Fan-Browder: minimax theorems, the Nash equilibrium theorem, the Gale-Nikaido-Debreu theorem, and the Ky Fan minimax inequality. Their study is based on and utilizes the techniques of simplicial structure and the FanBrowder map. Recall that for any abstract convex spaces satisfying abstract KKM principle we can deduce such classical theorems without using any Infimum Principles. Moreover, we note that the newly defined $L^{*}$-spaces in [45] are particular types of abstract convex spaces satisfying the abstract KKM principle.

(XII) In 2008, for $\mathbb{B}$-spaces, Briec and Horvath [30] showed that some theorems mentioned in this paper hold, that is, Fan-Browder fixed point theorem, Himmelbergtype (in fact, Browder-type and Kakutani-type) fixed point theorems, Fan type minimax inequality, existence of Nash equilibria, and others. Note that $\mathbb{B}$-spaces are KKM spaces [30, Corollary 2.2], and their authors depend the Pelegs type multiple KKM theorem.

(XIII) Finally, recall that there are several hundred published works on the KKM theory and we can cover only a part of them. For more historical background for the related fixed point theory and for more involved or related results in this paper, see the references [24-27, 41, 43, 48] and the literature therein.

\section{References}

[1] J. von Neumann, "Zur theorie der gesellschaftsspiele," Mathematische Annalen, vol. 100, no. 1, pp. 295-320, 1928.

[2] J. von Neumann, "Über ein ökonomisches Gleichungssystem und eine Verallgemeinerung des Brouwerschen Fixpunktsatzes," Ergebnisse eines Mathematischen Kolloquiums, vol. 8, pp. 73-83, 1937.

[3] S. Kakutani, "A generalization of Brouwer's fixed point theorem," Duke Mathematical Journal, vol. 8, pp. 457-459, 1941.

[4] J. F. Nash Jr., "Equilibrium points in N-person games," Proceedings of the National Academy of Sciences of the United States of America, vol. 36, pp. 48-49, 1950.

[5] J. Nash, "Non-cooperative games," Annals of Mathematics, vol. 54, pp. 286-295, 1951.

[6] K. Fan, "Fixed-point and minimax theorems in locally convex topological linear spaces," Proceedings of the National Academy of Sciences of the United States of America, vol. 38, pp. 121-126, 1952.

[7] I. L. Glicksberg, "A further generalization of the Kakutani fixed theorem, with application to Nash equilibrium points," Proceedings of the American Mathematical Society, vol. 3, pp. 170-174, 1952.

[8] C. J. Himmelberg, "Fixed points of compact multifunctions," Journal of Mathematical Analysis and Applications, vol. 38, pp. 205-207, 1972.

[9] K. Fan, "A generalization of Tychonoff's fixed point theorem," Mathematische Annalen, vol. 142, pp. 305-310, 1961.

[10] K. Fan, "Sur un théorème minimax," Comptes Rendus des Séances de l'Académie des Sciences. Série I, vol. 259, pp. 3925-3928, 1964.

[11] K. Fan, "Applications of a theorem concerning sets with convex sections," Mathematische Annalen, vol. 163, pp. 189-203, 1966. 
[12] T. Ma, "On sets with convex sections," Journal of Mathematical Analysis and Applications, vol. 27, pp. 413-416, 1969.

[13] A. Granas, "Sur quelques méthodes topologiques én analyse convexe," in Méthodes Topologiques en Analyse Convexe, vol. 110, pp. 11-77, Presses Université de Montréal, Montreal, Canada, 1990.

[14] A. Idzik and S. Park, "Leray-Schauder type theorems and equilibrium existence theorems," in Differential Inclusions and Optimal Control, vol. 2 of Lecture Notes in Nonlinear Analysis, pp. 191-197, University in Torun, Torun, Poland, 1998.

[15] S. Park, "Applications of the Idzik fixed point theorem," Nonlinear Functional Analysis and Its Applications, vol. 1, pp. 21-56, 1996.

[16] S. Park, "Remarks on a social equilibrium existence theorem of G. Debreu," Applied Mathematics Letters, vol. 11, no. 5, pp. 51-54, 1998.

[17] S. Park, "Minimax theorems and the Nash equilibria on generalized convex spaces," in NLA98: Convex Analysis and Chaos, vol. 1 of Josai Mathematical Monographs, pp. 33-45, Josai University, Sakado, Japan, 1999.

[18] S. Park, "Elements of the KKM theory for generalized convex spaces," The Korean Journal of Computational \& Applied Mathematics, vol. 7, no. 1, pp. 1-28, 2000.

[19] S. Park, "Fixed points, intersection theorems, variational inequalities, and equilibrium theorems," International Journal of Mathematics and Mathematical Sciences, vol. 24, no. 2, pp. 73-93, 2000.

[20] S. Park, "Acyclic versions of the von Neumann and Nash equilibrium theorems," Journal of Computational and Applied Mathematics, vol. 113, no. 1-2, pp. 83-91, 2000.

[21] S. Park, "New topological versions of the Fan-Browder fixed point theorem," Nonlinear Analysis: Theory, Methods E Applications, vol. 47, no. 1, pp. 595-606, 2001.

[22] S. Park, "Generalizations of the Nash equilibrium theorem on generalized convex spaces," Journal of the Korean Mathematical Society, vol. 38, no. 4, pp. 697-709, 2001.

[23] S. Park, "Remarks on acyclic versions of generalized von Neumann and Nash equilibrium theorems," Applied Mathematics Letters, vol. 15, no. 5, pp. 641-647, 2002.

[24] S. Park, "Elements of the KKM theory on abstract convex spaces," Journal of the Korean Mathematical Society, vol. 45, no. 1, pp. 1-27, 2008.

[25] S. Park, "Equilibrium existence theorems in KKM spaces," Nonlinear Analysis: Theory, Methods $\mathcal{E}$ Applications, vol. 69, no. 12, pp. 4352-5364, 2008.

[26] S. Park, "New foundations of the KKM theory," Journal of Nonlinear and Convex Analysis, vol. 9, no. 3, pp. 331-350, 2008.

[27] S. Park, "From the KKM principle to the Nash equilibria," International Journal of Mathematics and Statistics, vol. 6, no. S10, pp. 77-88, 2010.

[28] S. Park and J. A. Park, "The Idzik type quasivariational inequalities and noncompact optimization problems," Colloquium Mathematicum, vol. 71, no. 2, pp. 287-295, 1996.

[29] R. Bielawski, "Simplicial convexity and its applications," Journal of Mathematical Analysis and Applications, vol. 127, no. 1, pp. 155-171, 1987.

[30] W. Briec and C. Horvath, "Nash points, Ky Fan inequality and equilibria of abstract economies in Max-Plus and BB-convexity," Journal of Mathematical Analysis and Applications, vol. 341, no. 1, pp. 188 199, 2008.

[31] L. González, S. Kilmer, and J. Rebaza, “From a KKM theorem to Nash equilibria in L-spaces," Topology and Its Applications, vol. 155, no. 3, pp. 165-170, 2007.

[32] W. A. Kirk, B. Sims, and G. X.-Z. Yuan, "The Knaster-Kuratowski and Mazurkiewicz theory in hyperconvex metric spaces and some of its applications," Nonlinear Analysis: Theory, Methods $\mathcal{E}$ Applications, vol. 39, no. 5, pp. 611-627, 2000.

[33] Q. Luo, "KKM and Nash equilibria type theorems in topological ordered spaces," Journal of Mathematical Analysis and Applications, vol. 264, no. 2, pp. 262-269, 2001.

[34] B. Knaster, K. Kuratowski, and S. Mazurkiewicz, "Ein beweis des fixpunktsatzes für n-dimensionale simplexe," Fundamenta Mathematicae, vol. 14, pp. 132-137, 1929.

[35] M. Lassonde, "On the use of KKM multifunctions in fixed point theory and related topics," Journal of Mathematical Analysis and Applications, vol. 97, no. 1, pp. 151-201, 1983.

[36] C. D. Horvath, "Contractibility and generalized convexity," Journal of Mathematical Analysis and Applications, vol. 156, no. 2, pp. 341-357, 1991.

[37] C. D. Horvath, "Extension and selection theorems in topological spaces with a generalized convexity structure," Toulouse Annales Faculté des Sciences, vol. 2, no. 2, pp. 253-269, 1993.

[38] S. Reich and I. Shafrir, "Nonexpansive iterations in hyperbolic spaces," Nonlinear Analysis: Theory, Methods \& Applications, vol. 15, no. 6, pp. 537-558, 1990. 
[39] C. D. Horvath and J. V. Llinares Ciscar, "Maximal elements and fixed points for binary relations on topological ordered spaces," Journal of Mathematical Economics, vol. 25, no. 3, pp. 291-306, 1996.

[40] S. Park, "Continuous selection theorems in generalized convex spaces," Numerical Functional Analysis and Optimization, vol. 20, no. 5-6, pp. 567-583, 1999.

[41] S. Park, "Ninety years of the Brouwer fixed point theorem," Vietnam Journal of Mathematics, vol. 27, no. 3, pp. 187-222, 1999.

[42] S. Park, "Fixed points of better admissible maps on generalized convex spaces," Journal of the Korean Mathematical Society, vol. 37, no. 6, pp. 885-899, 2000.

[43] S. Park, "Generalized convex spaces, L-spaces, and FC-spaces," Journal of Global Optimization, vol. 45, no. 2, pp. 203-210, 2009.

[44] P. Q. Khanh, N. H. Quan, and J.-C. Yao, "Generalized KKM-type theorems in GFC-spaces and applications," Nonlinear Analysis: Theory, Methods \& Applications, vol. 71, no. 3-4, pp. 1227-1234, 2009.

[45] W. Kulpa and A. Szymanski, "Applications of general infimum principles to fixed-point theory and game theory," Set-Valued Analysis, vol. 16, no. 4, pp. 375-398, 2008.

[46] W. A. Kirk and B. Panyanak, "Best approximation in R-trees," Numerical Functional Analysis and Optimization, vol. 28, no. 5-6, pp. 681-690, 2007.

[47] C. D. Horvath, "Topological convexities, selections and fixed points," Topology and Its Applications, vol. 155, no. 8, pp. 830-850, 2008.

[48] S. Park, "Remarks on the partial KKM principle," Nonlinear Analysis Forum, vol. 14, pp. 51-62, 2009.

[49] K. Fan, "A minimax inequality and applications," in Inequalities, III, O. Shisha, Ed., pp. 103-113, Academic Press, New York, NY, USA, 1972.

[50] Y. J. Cho, J. K. Kim, and B. Y. Lee, "Remarks on KKM maps and applications," The Journal of Advanced Research in Applied Mathematics, vol. 1, no. 1, pp. 1-8, 2009.

[51] F. C. Liu, "A note on the von Neumann-Sion minimax principle," Bulletin of the Institute of Mathematics. Academia Sinica, vol. 6, no. 2, part 2, pp. 517-524, 1978.

[52] M. H. Shih and K.-K. Tan, "Noncompact sets with convex sections. II," Journal of Mathematical Analysis and Applications, vol. 120, no. 1, pp. 264-270, 1986.

[53] H. Nikaidô, “On von Neumann's minimax theorem," Pacific Journal of Mathematics, vol. 4, pp. 65-72, 1954.

[54] M. Sion, “On general minimax theorems," Pacific Journal of Mathematics, vol. 8, pp. 171-176, 1958.

[55] H. Komiya, "Convexity on a topological space," Fundamenta Mathematicae, vol. 111, no. 2, pp. 107-113, 1981.

[56] S. Park, "Foundations of the KKM theory via coincidences of composites of upper semicontinuous maps," Journal of the Korean Mathematical Society, vol. 31, no. 3, pp. 493-519, 1994.

[57] S. Simons, "Two-function minimax theorems and variational inequalities for functions on compact and noncompact sets, with some comments on fixed-point theorems," in Nonlinear Functional Analysis and Its Applications, Part 2, vol. 45 of Proceedings of Symposia in Pure Mathematics, pp. 377-392, American Mathematical Society, Providence, RI, USA, 1986.

[58] E. Tarafdar, "Fixed point theorems in $H$-spaces and equilibrium points of abstract economies," Journal of Australian Mathematical Society, vol. 53, no. 2, pp. 252-260, 1992.

[59] S. Y. Chang, "A generalization of KKM principle and its applications," Soochow Journal of Mathematics, vol. 15 , no. 1 , pp. $7-17,1989$.

[60] H. Nikaidô and K. Isoda, "Note on non-cooperative convex games," Pacific Journal of Mathematics, vol. 5, pp. 807-815, 1955.

[61] K.-K. Tan, J. Yu, and X.-Z. Yuan, “Existence theorems of Nash equilibria for non-cooperative N-person games," International Journal of Game Theory, vol. 24, no. 3, pp. 217-222, 1995.

[62] S. Park, "Variational inequalities and extremal principles," Journal of the Korean Mathematical Society, vol. 28, no. 1, pp. 45-56, 1991.

[63] S. Park and S. K. Kim, "On generalized extremal principles," Bulletin of the Korean Mathematical Society, vol. 27, no. 1, pp. 49-52, 1990.

[64] H. F. Bohnenblust and S. Karlin, "On a theorem of Ville," in Contributions to the Theory of Games, Annals of Mathematics Studies, no. 24, pp. 155-160, Princeton University Press, Princeton, NJ, USA, 1950.

[65] G. B. Dantzig, "Constructive proof of the min-max theorem," Pacific Journal of Mathematics, vol. 6, pp. 25-33, 1956.

[66] G. Debreu, "A social equilibrium existence theorem," Proceedings of the National Academy of Sciences of the United States of America, vol. 38, pp. 886-893, 1952.

[67] M. Lassonde, "Fixed points for Kakutani factorizable multifunctions," Journal of Mathematical Analysis and Applications, vol. 152, no. 1, pp. 46-60, 1990. 
[68] J. P. Torres-Martínez, "Fixed points as Nash equilibria," Fixed Point Theory and Applications, vol. 2006, Article ID 36135, 4 pages, 2006.

[69] H. Ben-El-Mechaiekh, P. Deguire, and A. Granas, "Une alternative non linéaire en analyse convexe et applications," Comptes Rendus des Séances de l'Académie des Sciences. Série I, vol. 295, no. 3, pp. 257-259, 1982.

[70] F. E. Browder, "The fixed point theory of multi-valued mappings in topological vector spaces," Mathematische Annalen, vol. 177, pp. 283-301, 1968.

[71] G. L. Cain Jr. and L. González, "The Knaster-Kuratowski-Mazurkiewicz theorem and abstract convexities," Journal of Mathematical Analysis and Applications, vol. 338, no. 1, pp. 563-571, 2008.

[72] M. A. Khamsi, "KKM and Ky Fan theorems in hyperconvex metric spaces," Journal of Mathematical Analysis and Applications, vol. 204, no. 1, pp. 298-306, 1996.

[73] S. Eilenberg and D. Montgomery, "Fixed point theorems for multi-valued transformations," American Journal of Mathematics, vol. 68, pp. 214-222, 1946. 